\title{
The cellular roles of the lissencephaly gene LIS1, and what they tell us about brain development
}

\author{
Richard B. Vallee ${ }^{1}$ and Jin-Wu Tsai \\ Department of Pathology and Cell Biology, Center for Neurobiology and Behavior, Columbia University College of \\ Physicians and Surgeons, New York, New York 10032 USA
}

Lissencephaly is a severe brain developmental disease characterized by mislocalization of cortical neurons. This class of diseases has received considerable recent attention for their promise in shedding light on the mechanisms underlying the massive waves of neural progenitor cell migration required for brain development. Classical lissencephaly results from sporadic mutations in the human LIS1 gene, which has been implicated in regulating the microtubule motor protein cytoplasmic dynein. This connection supports a role for LIS1 in cell motility, and suggests that defects in dyneinmediated movement are responsible for lissencephaly. This article reviews evidence regarding the cellular defects contributing to classical lissencephaly, with emphasis on recent live analysis of LIS1-deficient neural progenitor cell motility in brain tissue.

\section{Lissencephaly}

The lissencephalopathies are brain developmental diseases characterized by decreased cortical complexity (Crome 1956; Barkovich et al. 1991; Dobyns and Truwit 1995). The surface of the brain is relatively smooth, giving rise to the name of the condition, which translates from Greek to "smooth brain." Classical, or type I lissencephaly, is caused by sporadic mutations in the LIS1 gene (Reiner et al. 1993; Lo Nigro et al. 1997). X-linked lissencephaly is caused by both familial and sporadic mutations in the DCX gene, which encodes the protein doublecortin (des Portes et al. 1998; Gleeson et al. 1998).

These diseases have received considerable attention during the past half decade for a number of reasons. Brain size is generally greater in the lissencephalopathies than in microcephalic conditions. This distinction suggests that progenitor cell proliferation plays less of a role in lissencephaly. Differentiated neurons are produced, but many are mislocalized, typically in a broad ectopic lamina internal to the normal cortical layers (Stewart et

${ }^{1}$ Corresponding author.

E-MAIL rv2025@columbia.edu; FAX (212) 305-549.

Article is online at http://www.genesdev.org/cgi/doi/10.1101/gad.1417206. al. 1975). Together these observations have suggested that LIS1 and DCX may play roles in neural progenitor cell migration, a very important but somewhat mysterious aspect of brain development in which only a few genes have been identified.

Both LIS1 and doublecortin are now known to participate in microtubule-based behavior. Doublecortin is a microtubule-binding and stabilizing protein (Gleeson et al. 1999). Why mutations in DCX lead to lissencephaly, whereas mutations in the many other known MAPs (microtubule-associated proteins) have not been associated with this disease is uncertain. LIS1 is involved in regulating a microtubule motor protein, cytoplasmic dynein (Faulkner et al. 2000; Sasaki et al. 2000; Smith et al. 2000). In this case, microtubule-dependent cell motility is involved, as evidenced by live imaging of LIS1-deficient neural progenitor cells (Tsai et al. 2005).

This perspective is focused on the cellular functions of LIS1, with emphasis on its molecular interactions and its role in neural progenitor cell behavior. We argue on the basis of studies with neural progenitors as well as nonneuronal cells that classical lissencephaly results from defects in cytoplasmic dynein function, involving not only cell migration but also division and morphogenesis. Analysis of LIS1 is already leading to new insight into the complex cell biology of neural progenitors, an issue of broad importance for understanding normal and abnormal brain development, and potentially the behavior of stem cell in repopulating neuronal tissue.

\section{LIS1 interactions}

LIS1 has been identified in two biological pathways (Fig. 1). The LIS1 polypeptide was initially identified as a stoichiometric component of purified platelet-activating factor acetyl-hydrolase (PAFAH)-1b (Hattori et al. 1994), an enzyme involved in deactivation of the lipid messenger platelet-activating factor. Mice null for the catalytic subunits of PAFAH-1b showed no apparent brain developmental phenotype (Koizumi et al. 2003; Yan et al. 2003), however, suggesting that some other LIS1 function is responsible for lissencephaly. An ortholog of LIS1, NudF, 


\section{Dynein/LIS1 Interactions}

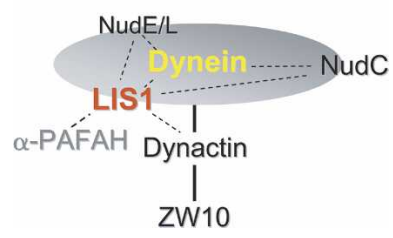

Figure 1. Interactions involving LIS1 and cytoplasmic dynein. LIS1 interacts with PAFAH and cytoplasmic dynein, but only the latter is involved in lissencephaly. LIS1 interacts with three sites within cytoplasmic dynein itself, and a fourth site within the dynactin complex, which is involved in subcellular targeting of dynein through ZW10 and other mechanisms. LIS1 and cytoplasmic dynein both interact with NudE and its isoform NudEL, and with NudC. The effect of these interactions on cytoplasmic dynein activities remains uncertain.

was independently identified as a regulator of cytoplasmic dynein function in the filamentous fungus Aspergillus nidulans (Xiang et al. 1995). Mutations in NudF resulted in the same phenotype observed for cytoplasmic dynein, failure of nuclei to distribute throughout the hyphal processes. NudF did not exhibit a readily detectable interaction with cytoplasmic dynein in this system, in contrast to the integral association of LIS1 with vertebrate PAFAH- $1 b$.

LIS1 does, in fact, bind to vertebrate cytoplasmic dynein as judged using several assays (Faulkner et al. 2000; Sasaki et al. 2000; Smith et al. 2000; Tai et al. 2002). The interaction is substoichiometric, and occurs through multiple sites. LIS1 interacts with three distinct sites within dynein itself, including the basal stem or tail portion of the molecule responsible for cargo binding and the motor domain (Tai et al. 2002). LIS1 also interacts with the dynamitin subunit of dynactin, a dynein accessory complex that interacts with dynein and mediates its attachment to diverse subcellular structures. Aspergillus $N u d F$ was found to interact with the NudE (Efimov and Morris 2000) and NudC genes. Vertebrate LIS1 was subsequently found to associate physically with two NudE orthologs, mNudE (Kitagawa et al. 2000) and its isoform NudEL (Niethammer et al. 2000), and with mNudC (Morris et al. 1998). Each of these proteins, in turn, interacts with cytoplasmic dynein (Sasaki et al. 2000; Aumais et al. 2001). Additional LIS1, dynein, and dynactin interactors, such as CLIP-170 (Coquelle et al. 2002; Komarova et al. 2002; Tai et al. 2002), have been identified, as have numerous binding partners of NudE and NudEL (Feng et al. 2000; Morris et al. 2003; Toyo-oka et al. 2003; Liang et al. 2004).

The physiological requirement for this degree of complexity is poorly understood. Cytoplasmic dynein is a mechanochemical enzyme, and NudEL and LIS1 each associate with the dynein motor domain. Thus, it is appealing to speculate that much of the complexity in dynein composition and interactions relates to the fine control of dynein activity. In addition, cytoplasmic dynein participates in numerous cellular activities associated at diverse sites within the cell. Some functions involve transport of membranous organelles or macromolecule complexes, but others appear to involve tension generation. This range of roles may, quite conceivably, also require a highly complex range of regulatory factors and mechanisms. Although it has been reported that LIS1 causes some stimulation of dynein ATPase activity (Mesngon et al. 2006), very little is understood regarding the role of LIS1 and its binding partners in regulation of dynein motor and cargo-binding activities. Considerably more has been determined regarding the range of subcellular functions in which LIS1 participates.

\section{Physiological roles of LIS1 and cytoplasmic dynein}

As noted above, both cytoplasmic dynein and LIS1 have been implicated in nuclear movement by phenotypic analysis in Aspergillus. The underlying mechanism by which the motor protein moves nuclei in this system is not completely understood. Dynein and the LIS1 ortho$\log \mathrm{NudF}$ have each been localized to the tips of microtubules in this system (Han et al. 2001), but their role there is uncertain. Dynein has also been detected at the tips of growing hyphae (Beckwith and Morris 1995). From this site, dynein, regulated by LIS1, would be appropriately positioned to pull on microtubules emanating from their nucleation site at the nuclear surface, the pole body. This mechanism may indeed contribute to movement of the most distally situated nuclei toward the hyphal tip. However, it is unlikely to account for movement of nuclei within the rest of the long hyphal process. These movements are bidirectional and serve to space nuclei from each other to produce a relatively uniform distribution along the hypha. How LIS1 and dynein might serve in bidirectional movement is unclear. Conceivably, dynein might be distributed in a disordered array throughout the cortex of the cell (Dujardin et al. 2003; see below), from which site it might pull on microtubules. As in in vitro microtubule gliding on cytoplasmic dynein monolayers (Paschal and Vallee 1987; Paschal et al. 1987), the direction of force production would be defined solely by the orientation of the microtubules, which may be the case for microtubules emanating from the pole bodies in Apsergillus hyphae. LIS1, along with cytoplasmic dynein, have been implicated in nuclear transport or positioning in other systems as well. In Drosophila, LIS1 was reported to participate along with dynein in nuclear positioning within the oocyte (Liu et al. 1999; Lei and Warrior 2000) and in the yeast Saccharomyces cerevisiae in the proper orientation of the dividing nucleus to enter the bud (Eshel et al. 1993; Li et al. 1993; Lee et al. 2003).

The functions of LIS1 have also been studied in vertebrate cultured cells, in which dynein is involved in a greater variety of roles. LIS1 was found to colocalize with cytoplasmic dynein at mitotic kinetochores, another result consistent with a role for LIS1 in the dynein pathway and in chromosome behavior in particular (Faulkner 
et al. 2000; Tai et al. 2002). Overexpression of full-length LIS1 or its $\mathrm{N}$ - and $\mathrm{C}$-terminal domains caused a very pronounced increase in mitotic index, potentially a sign of mitotic delay. Microinjection of an anti-LIS1 antibody into dividing cells indeed delayed mitotic progression (Faulkner et al. 2000). Consistent with a role for LIS1 at kinetochores, chromosomes exhibited an unusual defect in alignment at the metaphase plate. Cells injected with an anticytoplasmic dynein antibody showed a related but more severe alignment defect (Faulkner et al. 2000).

LIS1 was also found to localize to the cortex of dividing epithelial cells (Faulkner et al. 2000), a site at which cytoplasmic dynein had only recently been observed (Busson et al. 1998). Overexpression of LIS1 resulted in misalignment of the mitotic spindle along with disruption of the cortical dynein distribution pattern. These results suggested that dynein, along with LIS1, serves to regulate spindle orientation. Mitotic cleavage plane has been proposed to be important in the timing of neurogenesis within the neuroepithelial layer in the developing ventricular zone (for review, see Gotz and Huttner 2005). The LIS1 phenotypic data together, therefore, predicted several potential defects in the LIS1 heterozygous brain: interference with mitotic progression, altered spindle orientation, and consequent defects in the timing of neurogenesis (Faulkner et al. 2000).

That cell division defects might be important in vivo has received some support, albeit indirect, from analysis of mutant mouse and fly models. In hypomorphic mutant dLIS1 fruit flies, ovarian cell number was reduced (Swan et al. 1999). Homozygous null LIS1 mice exhibited preimplantation embryonic lethality (Hirotsune et al. 1998), consistent with a basic and general cellular role for LIS1. However, in LIS1 hemizygous mice, effects on cell division within the developing brain were limited (Gambello et al. 2003). Intriguingly, BrdU-positive cells were somewhat increased near the ventricular surface, but declined to below normal levels deeper into the developing neocortex.

Another site of cytoplasmic dynein and LIS1 colocalization is the centrosome (Sasaki et al. 2000; Smith et al. 2000), the major organizing center for microtubules in undifferentiated cells. However, the functional implications of this aspect of LIS1 and dynein distribution are uncertain. The centrosome nucleates microtubule assembly and represents a site at which microtubule minus ends converge. It is toward the minus ends that cytoplasmic dynein produces force. To an extent, therefore, the presence of dynein and LIS1 at the centrosome may represent the accumulation of transported material. However, dynein and LIS1 concentrate at centrosomes even in the absence of microtubules, and potential targeting proteins for dynein have been identified at the centrosome (Feng et al. 2000; Uetake et al. 2004). These observations suggest that dynein has additional functions at the centrosome itself. One intriguing possibility is that centrosomal dynein acts as a plus-end-directed microtubule transporter. Dynein has, in fact, been implicated in the outward transport of noncentrosomeal microtubules (Ahmad et al. 1998; Abal et al. 2002). Con- ceivably, centrosome-associated dynein could serve to initiate microtubule transport. We suggest that such behavior could also serve to correct anomalous protrusion of microtubule minus ends from the centrosomal region. Whether this type of microtubule misbehavior occurs in cells is not known.

Complicating the issue of a centrosomal dynein function, dynein and LIS1 do have clear roles in the positioning of the centrosome within the cell (Palazzo et al. 2001) (Etienne-Manneville and Hall 2001; Burakov et al. 2003; Dujardin et al. 2003; Vallee and Stehman 2005). We argue, however, that this important function reflects a role for dynein elsewhere in the cell; i.e., at the cortex (Burakov et al. 2003; Dujardin et al. 2003; Vallee and Stehman 2005). As an early step in wound-healing assays, centrosomes become reoriented toward the leading edge of the cell as it prepares to migrate to close the gap (Palazzo et al. 2001). This behavior does not appear to involve movement of the centrosome itself, which instead is now known simply to remain at the cell center while the nucleus and other subcellular structures are pushed toward the rear by actomyosin-dependent forces (Gomes et al. 2005). Together, these data suggest that cytoplasmic dynein pulls on the centrosome from the wound edge; i.e., the direction of migration. In fact, immunocytochemical analysis of wounded monolayers revealed that cytoplasmic dynein, its companion complex dynactin, and LIS1 all become concentrated at the leading cell edge under these conditions (Dujardin et al. 2003). The proteins are found as small puncta both arrayed along microtubules and at their tips, but mostly between and beyond the reach of the leading microtubules. These observations suggest that dynein associates independently with the cell cortex. Recent work has revealed that ZW10, a prominent dynein anchoring protein at the kinetochore, also becomes redistributed to the leading cortex in migrating cells (Varma et al. 2006), suggesting substantial similarity between anchoring mechanisms at distinct subcellular sites of dynein and LIS1 activity.

Together, these results argue that centrosome position serves as a useful marker for dynein activity in migrating cells, presumably involving that subfraction of dynein associated with the leading cell cortex. The contribution of the centrosome-associated dynein pool to migration remains much less well understood.

It should be noted that the redistribution of dynein and LIS1 to the leading cortex persists throughout active wound healing (Dujardin et al. 2003) well after centrosome/nuclear reorientation has occurred. This observation suggested that dynein, dynactin, and LIS1 function in more than the initial aspects of directed migration. Indeed, inhibition of dynein, LIS1, or dynactin by use of antibody or dominant negative cDNA injection each reduced the forward migration rate of cells at the wound edge by about half. An equivalent effect was produced in cells injected with antidynein antibody following the initial 2-h period of wound healing, during which centrosome reorientation had been completed. This and other results support an independent role for dynein and its regulatory factors in forward migration of the whole 
cell. The specific role of these proteins in this activity is not understood, but we suspect that it involves local activation of lamellipodial activity by a dynein-mediated tension-sensing mechanism (Dujardin et al. 2003).

Finally, as an apparent cytoplasmic dynein regulator, LIS1 might be expected to participate in minusend-directed vesicular transport. Surprisingly, dominant negative LIS1 cDNAs had no clear effect on Golgi, endosome, and lysosome distribution (Faulkner et al. 2000; Tai et al. 2002). These results raise the interesting possibility that LIS1 may regulate only a subset of dynein functions and may not participate in retrograde axonal transport in particular.

\section{Role of LIS1 in neural progenitor cell behavior}

To evaluate the roles of LIS1 and dynein in neural progenitor cell behavior directly, in utero electroporation of fetal rat brain has been employed using a variety of reagents. These include plasmids encoding short hairpin RNAs (shRNAs) (Shu et al. 2004; Tsai et al. 2005), dominant negative LIS1 and dynactin cDNAs already characterized in in vitro studies (see above), and synthetic LIS1 small inhibitory RNAs (siRNAs) (Tsai et al. 2005). Each of these reagents affected the overall redistribution of neural progenitors as evidenced by the accumulation of transfected cells, especially in the subventricular zone. A similar study involving the doublecortin gene has produced a comparable accumulation of cells within the subventricular zone (Bai et al. 2003) consistent with a related cellular origin for lissenceaphalies involving different genetic causes.

In order to investigate the roles of LIS1 and dynein at the cellular and subcellular level, we have cultured slices from transfected brains and monitored them by timelapse fluorescence microscopy (Tsai et al. 2005). For these studies, a vector-based RNA interference (RNAi) approach was most useful. Among other advantages, plasmids can be used that encode GFP along with the LIS1 shRNA, allowing individual experimental cells to be readily distinguished from the surrounding nontransfected cells. Processes from individual cells can also be traced over long distances, and the fates of individual cells and their processes could be followed for several days. The behavior of cells examined in this way is complex, but reveals their entire early course of development (Fig. 2). The transfected DNA is taken up by radial glial cells. These cells extend long processes to both the ventricular and pial surfaces of the developing neocortex, and DNA uptake is presumed to occur only through their small "endfeet" (or apical membrane) at the ventricular surface. Proliferation occurs through symmetric mitotic divisions and migratory neural cells are generated through asymmetric divisions. Then, through a poorly monitored portion of the migratory pathway, the nascent neural cells ascend to the subventricular zone. They now exhibit multiple short processes, including a single apparent axon that continues to elongate through subsequent developmental stages. A subpopulation of multipolar cells continue to divide, albeit at a low rate.

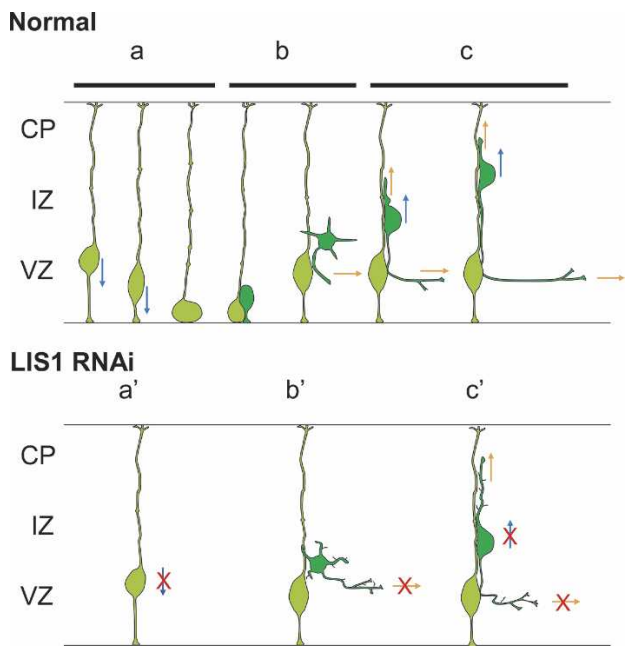

Figure 2. Pathway for neural progenitor cell morphogenesis and migration in the fetal neocortex. Proliferating radial glial cells expand through symmetric divisions, and generate neurons through assymetric divisions $(a)$, which ascend to the subventricular zone, where they reside as multipolar cells $(b) .(c)$ The latter transform into bipolar cells, which locomote along radial glial fibers toward the pial surface of the developing cortex (Noctor et al. 2004). In cells subjected to LIS1 RNAi, these stages remain readily recognizable. $\left(a^{\prime}\right)$ However, LIS1 RNAi arrests the interkinetic nuclear oscillations characteristic of radial glial cells, as well as their mitotic divisions. $\left(b^{\prime}\right)$ Many cells, nonetheless, reach the multipolar stage, though the transformation to the bipolar state is blocked, as is the extension of axonal processes. Those few cells that reach the intermediate zone have a characteristic bipolar morphology. $\left(c^{\prime}\right)$ Nonetheless, radial migration of the cell soma is arrested, though the forward extension of their migratory processes persists. (VZ) Ventricular zone; (SVZ) subventricular zone; (IZ) intermediate zone; (CP) cortical plate. Modified with permission from Tsai et al. (2005). (Reproduced from The Journal of Cell Biology by copyright permission of The Rockefeller University Press.)

After about a day, these cells emerge from the subventricular zone with a bipolar morphology, at which point they can be seen to migrate directionally along radial glial fibers through a process known as locomotion.

Time-lapse analysis of cells subjected to vector-based LIS1 RNAi exhibited defects at each stage of the neuronal migration pathway (Tsai et al. 2005). This result was surprising in view of the pronounced accumulation of cells, mainly within the subventricular zone. It appears to reflect a nonuniformity in transfection efficiency among neural progenitor cells, superimposed on a discontinuous pathway for neuronal migration in which LIS1 participates.

\section{Radial glial cells}

Transfected radial glial cells could be identified by their highly elongated processes, which could be seen to extend to the ventricular and pial surfaces of the developing brain. The nuclei showed clear oscillatory behavior, another well-established characteristic. The movements were very discontinuous, with pauses of several hours separating directed migration events. In cells subjected 
to LIS1 RNAi, the directed nuclear movements were abolished. The nuclei were arrested at random positions relative to the ventricular surface, with no apparent accumulation at a particular position within the cell. The cells retained their normal morphology, arguing against an indirect effect of LIS1 RNAi on the substructure of the cell, and for a specific role for LIS1 in nuclear transport (nucleokinesis).

\section{Multipolar subventricular zone cells}

As noted above, interference with LIS1 expression or function using LIS1 siRNAs, vector-based RNAi, and dominant cDNAs each resulted in a marked accumulation of transfected cells in the subventricular zone. A similar pattern was observed for cells transfected with an RNAi construct for a cytoplasmic dynein subunit (S.C. Tan, J.-W. Tsai, and R.B. Vallee, unpubl.) and the dominant negative dynamitin subunit of dynactin (Tsai et al. 2005). In the case of vector-based LIS1 RNAi, this observation was puzzling in view of the complete arrest in nucleokinesis in radial glial cells observed by live imaging (see above). A likely explanation for these paradoxical findings relates to the random character of the transfection method. In all likelihood, the arrested radial glial cells took up the highest concentration of LIS1 RNAi construct, leading to sufficiently rapid loss of LIS1 to lead to total arrest. Cells that reached a range of further stages in the migration pathway may represent those that lost LIS1 at progressively slower rates.

Cells that accumulated within the subventricular zone as a result of LIS1 RNAi or other treatments retained their multipolar morpology. This observation suggested that it might be the normal transition from the multipolar to bipolar migratory state that is a LIS1-dependent step. Time-lapse imaging was again used to evaluate this possibility. Control cells typically exhibited multiple processes with an average lifetime of $\sim 120$ min. Among the processes, a single, fine axon could be discerned, which grew at a constant rate of $\sim 1.2 \mu \mathrm{m} / \mathrm{min}$. Occasional cells were observed to undergo a dramatic morphological change to the migratory bipolar form with a major leading migratory process and a trailing axon. The transformation was rapid, occurring over $\sim 60$ min, and appeared to involve expansion of one of the existing processes as the others retracted. The conversion events were infrequent, consistent with the $\sim 1$-d lifetime for the multipolar state described previously.

In cells subjected to LIS1 RNAi, the transition from the multipolar to the bipolar state was abolished. Although overall cell morphology was preserved, some changes in the behavior of the multiple processes were evident. Process lifetime was similar, but process branching was increased by threefold. Axons persisted, but their elongation was arrested. That the latter structures formed at all suggests that they were initiated early during LIS1 RNAi. Further extension must have ceased once LIS1 levels had fallen sufficiently, but retraction was not observed during the period of observation (up to $\sim 1 \mathrm{~d}$ ). Together these results suggest that LIS1 is not required for axon maintenance, but rather, for axon elongation.
Bipolar cells in the subventricular and intermediate zones

A few GFP-positive bipolar cells could be observed to have reached the intermediate zone despite the block in conversion from the multipolar state. Again, the observed bipolar cells presumably formed before LIS1 depletion had become severe. Despite the mostly normal morphology of the bipolar cells, somal transport was blocked. Remarkably, however, the migratory processes continued to elongate, with process extension proceeding at almost the same rate as in control bipolar cells. Thus, the overall effect was complete uncoupling of somal movement from process growth.

The mechanism responsible for somal transport in these cells is incompletely understood. This form of motility is likely to represent another manifestation of nuclear migration within the cytoplasm. Its dependence on LIS1, which is clearly needed for oscillatory nucleokinesis within radial glial cells, provides a potentially important clue that this may be the case. A myosin II inhibitor has recently been reported to interfere with forward cell body movement in freely migrating bipolar neural progenitors (Bellion et al. 2005; Schaar and McConnell 2005). Potentially, the two motor systemsdynein-microtubule and myosin-actin-may work in concert in these cells. This possibility is in contrast to results obtained in fibroblasts. Cytoplasmic dynein has been implicated in pulling the nucleus forward, apparently by exerting tension on the microtubule network (see above), but myosin II was deduced to drive the nucleus and other cell contents to the rear (Gomes et al. 2005). The relationship between forces exerted on the nucleus in the two cell types remains to be more fully explored; in particular, how the sites where dynein acts in neural progenitors both at the bipolar and radial glial stages compare with the sites in fibroblasts remains a fascinating question.

The disparate effects of LIS1 RNAi on different classes of neural process suggest fundamental differences in their underlying organization. LIS1 RNAi had no apparent effect on growth of the migratory processes of bipolar cells while arresting axonal extension (Tsai et al. 2005). Recent evidence has revealed an accumulation of dynein and LIS1 in the growth cones of chick dorsal root and sympathetic axons treated with laminin to induce axon sprouting and rapid growth (P.W. Grabham, M. Bennecib, G.E. Seale, D.J. Goldberg, and R.B. Vallee, unpubl.). Inhibition of LIS1 or dynein using antibody injection blocked the laminin effects. Together with the in situ LIS1 RNAi data, these results suggest a specialized role for LIS1 and dynein in remodeling of the growth cone cytoskeleton that is not involved in the growth of migratory processes in bipolar progenitor cells.

\section{Role of LIS1 in division and proliferation of neural progenitors}

A long-standing question in brain developmental biology regards the relationship between the interkinetic kinetic 
nuclear oscillations that occur within radial glial cells and the timing of mitosis. This event occurs when the radial glial nuclei reach the ventricular surface, but whether division is regulated by position or simply correlated with it is unknown. In radial glial cells subjected to LIS1 RNAi, the cell body was arrested at a range of distances from the ventricular surface. None of these cells were observed to divide.

Conceivably, these results simply reflect a general role for LIS1 in mitotic entry. This possibility seems unlikely, however, in view of the effects of perturbing either LIS1 or dynein function in cultured cell lines. As noted above, LIS1 inhibition in nonneural cells caused a clear block during, but not prior to mitosis (Faulkner et al. 2000). It is possible that neural progenitors differ in their response to LIS1 inhibition, but this prospect is disputed by DAPI staining of brain sections from rats subjected to LIS1 RNAi (Tsai et al. 2005). As expected, in transfected radial glial cells, chromosome condensation was not observed in any of the nuclei situated at a distance from the ventricular surface, indicating that they were indeed in interphase. However, chromosomes were condensed in those few cells in which the nucleus had reached the ventricular surface. Whether these cells are delayed in mitosis is under investigation. However, we suspect that interference with LIS1 activity or reduced LIS1 expression may have two distinct effects, inhibition of progression through mitosis and uniquely in radial glial cells, inhibition of mitotic entry.

How mitotic entry in these cells is controlled is unknown, but it is likely to be controlled through a novel mechanism (Fig. 3). Because only those nuclei at the ventricular surface had entered $M$ phase, we imagine that a factor or factors required for mitosis may be sequestered

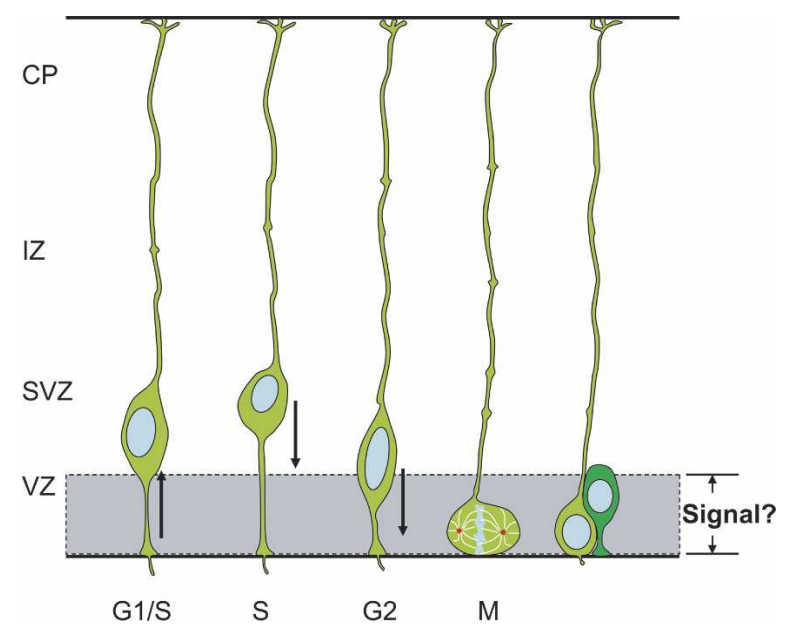

Figure 3. Cell cycle arrest in radial glial cells subjected to LIS1 RNAi. The cycle of interkinetic nuclear oscillations within radial glial cells is depicted. Cell cycle stage is indicated to correlate with distance from the ventricular surface. Absence of mitotic figures at the ventricular surface resulting from LIS1 RNAi may result from failure to reach an M-phase entry signal of unknown identity, proposed to be restricted to the ventricular surface. at this site. Such factors could be related to known elements whose asymmetric segregation during cell division may regulate neurogenesis. However, candidates for the latter function are not known to be involved in mitotic entry.

Finally, as noted above, LIS1 overexpression alters mitotic spindle orientation in polarized epithelial cell monolayers, in which mitosis normally occurs normal to the epithelial plane (Faulkner et al. 2000). Whether such an effect is also produced by reduced LIS1 expression and how it might affect proliferation and neurogenesis remain additional questions for further analysis.

We note that a spatial control model for the control of mitotic entry in radial glial cells makes excellent biological sense. The interkinetic nuclear oscillations are strikingly discontinuous. Although it has been difficult to gather extensive data on the complete time course of nuclear migration from one mitotic division to the next, it seems likely that the duration of this process must be quite variable. If so, temporal control of cell division by a cell-autonomous clock would be fraught with errors and characterized by a spread of mitotic figures over a relatively broad spatial range. Spatial control provides a simple way to avoid this outcome. This mechanism is likely to be specific to neural progenitors. Whether the identity of the controlling factors or just their sequestration mechanism are unique to these cells remain important questions for future study.

\section{Mechanisms for migration}

Although the complexity of the neuronal migration pathway has only recently become fully appreciated (for review, see Kriegstein and Noctor 2004), what is now referred to as locomotion of neural progenitors-the net displacement of the bipolar cell-has been a long-standing issue of interest in the field of developmental neurobiology. The recent studies on LIS1 have shed some light on a broader effort to understand the underlying mechanisms by which the bipolar progenitor cells move through the brain.

The movement of the cell body is known to be intermittent, but the significance of this observation has been unclear. Recent analysis of centrosome movement in cerebellar granule cells cocultured with radial glia has revealed a "two-stroke" mechanism for somal translocation (Fig. 4; Solecki et al. 2004). Using fluorescently tagged forms of dynamitin or mPar $6 \alpha$ as centrosome markers, this structure was observed to move forward independently of the nucleus. Following a delay, the nucleus caught up. This behavior was disrupted by overexpression of mPar $6 \alpha$, which altered microtubule and centrosome organization. A similar pattern of movement has been observed in freely migrating bipolar neural progenitor cells in vitro (Bellion et al. 2005; Schaar and McConnell 2005). In this case, a swelling appeared in the leading process prior to centrosome and nuclear movement (Schaar and McConnell 2005). Inhibition of myosin II using blebbistatin blocked somal movement. Together, these results have implicated both the micro- 


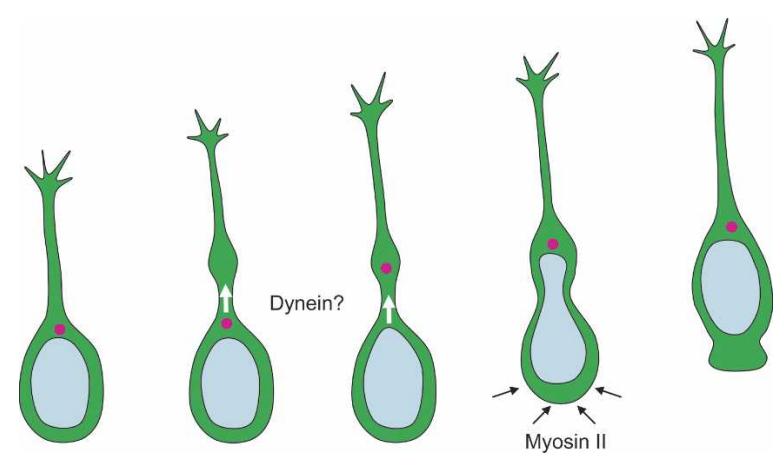

Figure 4. Migratory bipolar neural progenitor cell, showing just the nucleus and leading migratory process. Migration occurs discontinuously. A swelling first appears in advance of the nucleus. The centrosome then advances to this site, followed a short time later by the nucleus. As described in the text, LIS1 and cytoplasmic dynein have been implicated in the earlier steps based on the uncoupling of nuclear movement from process extension in live imaged neural progenitor cells subjected to RNAi. Reduction in LIS1, dynein, or NudEL expression increased the average separation between centrosome and nucleus. The myosin II inhibitor blebbistatin interferes with somal movement. These observations suggest important roles for cytoplasmic dynein and myosin II in migration, but their specific roles have not yet been fully elucidated.

tubule and the actomyosin networks in neural progenitor cell migration.

Reduction in LIS1 expression alone was sufficient to block somal movement in bipolar progenitor cells (Tsai et al. 2005). This observation implicates cytoplasmic dynein in nuclear movement, but which step in this process is involved is uncertain. LIS1 and cytoplasmic dynein may, quite reasonably, be expected to contribute to forward centrosome movement based on substantial evidence for regulation of centrosome position by dynein-mediated forces in other cell types (see above) (Etienne-Manneville and Hall 2001; Palazzo et al. 2001; Burakov et al. 2003; Dujardin et al. 2003; Gomes et al. 2005). Thus, inhibition of nucleokinesis in cells subjected to LIS1 RNAi could result from an effect on the initial step in the two-stroke process, the advance of the centrosome. Alternatively, or additionally, LIS1 may be involved in the subsequent step, the movement of the nucleus itself to catch up with the centrosome. These issues have not been addressed directly as yet. However, analysis of cerebellar granule cells from LIS1 heterozygous mice (Tanaka et al. 2004) and neocortical progenitor cells subjected to LIS1, NudEL, or dynein RNAi in situ (Shu et al. 2004) revealed small, but significant increases in the average spacing between nucleus and centrosome. How these results relate to the roles for LIS1 and dynein outlined here remains to be ascertained.

Where LIS1 and dynein act within neural progenitors remains a particularly important aspect of the migration problem. Finally, how the role of LIS1 and dynein in locomoting progenitor cells relates to that in radial glial cells will be important to address. A basic effect in cells at each developmental stage is to arrest nuclear move- ment. However, this process occurs bidirectionally in the radial glial cell, but unidirectionally in the bipolar cell. That the two cell forms may use a related mechanism for nucleokinesis is suggested by the results of LIS1 RNAi. The full extent to which the underlying mechanisms of motility overlap in these morphologically distinctive cells remains to be explored.

\section{Conclusion}

LIS1 has been a focus of recent research both for its value in understanding brain development and for its role in the etiology of lissencephaly itself. In addition to the characteristic smoothness of the cerebral surface, another hallmark of this class of diseases is a broad ectopic neuronal lamina internal to the normal cortical layers (Stewart et al. 1975). LIS1 and doublecortin RNAi have revealed that reduced expression in either of these genes causes formation of an accumulation of neural progenitors in the subventricular zone (Bai et al. 2003; Shu et al. 2004; Tsai et al. 2005). This feature of the RNAi phenotype seems very likely to explain why neurons are not randomly mispositioned, but rather accumulate in a particular cortical zone, albeit a broad one. Although neural progenitors are sensitive to reduced LIS1 at several stages in the migratory pathway, the ectopic neuronal lamina now seems likely to reflect a role for LIS1 in conversion of these cells from the nonmotile multipolar state to the bipolar migratory state (Tsai et al. 2005). Whether doublecortin regulates the same transition and how it and LIS1 contribute to this effect at the subcellular level remain to be explored further.

Lissencephaly is also accompanied by severe seizures and extreme loss of higher brain function. Whether the extent of neuronal mispositioning observed in lissencephalic children is sufficient to explain these dysfunctions is uncertain (Ross 2002). Defects in dendritic arborization have also been reported in LIS1 heterozygous mice (Fleck et al. 2000), which should also affect higher brain function. LIS1 RNAi has, in addition, shown defects in the outgrowth of axons, behavior that begins within the multipolar cells in the subventricular zone and continues throughout the subsequent radial migration of these cells after their conversion to a bipolar form (Tsai et al. 2005). Thus, it seems quite possible that some or many axons in the lissencephalic brain could fail to reach their targets. An important subject for further investigation will, therefore, be the extent of connectivity defects in the lissencephalic child and the degree to which they affect higher brain function.

The contribution of cell division defects to lissencephaly has been controversial. Although LIS1 has been implicated in cytoplasmic dynein-mediated mitotic events (Faulkner et al. 2000), it has been unclear how to interpret these effects in light of the modest effects of LIS1 haploinsufficiency on brain size in children and in mice (Sapir et al. 1997; Hirotsune et al. 1998; e.g., see Sweeney et al. 2000). LIS1 RNAi has produced the first direct evidence for LIS1-related defects in mitosis in vivo. However, the nature of the defects has been sur- 
prising. The most striking effect is a severe decrease in mitotic events within radial glial cells (Tsai et al. 2005). This effect appears to be unrelated to that observed in cultured nonneuronal cells, in which LIS1 or dynein inhibition cause a delay in progression through mitosis. However, the latter effects probably do occur, as evidenced by the persistence of some mitotic cells at the ventricular surface. We reason that the severity of this aspect of the LIS1 RNAi phenotype is greatly minimized by the extreme reduction in nuclei available to enter mitosis. Thus, absent the effect of LIS1 RNAi on nucleokinesis, we predict a severe accumulation of mitotic cells at the ventricular surface.

These effects must be translated with some caution to the lissencephalic brain, which is caused by reduction in active LIS1 of about half. We predict that mitotic entry events within radial glia will be reduced in frequency, but will persist. We predict that those cells that enter mitosis will be stalled, but will ultimately divide. The net result will, nonetheless, be a reduction in brain mass. However, histopathology of the lissencephalic brain will reveal complex effects on the distribution of cells at different cell cycle stages, as has been observed (Gambello et al. 2003).

Finally, LIS1 has emerged as a powerful tool for the dissection of brain developmental mechanisms in revealing what appears to be a long-hidden spatial control mechanism for mitotic entry. How and why this regulation occurs, and how it is related to other brain developmental problems remain fascinating questions for future study.

\section{References}

Abal, M., Piel, M., Bouckson-Castaing, V., Mogensen, M., Sibarita, J.B., and Bornens, M. 2002. Microtubule release from the centrosome in migrating cells. J. Cell Biol. 159: 731-737.

Ahmad, F.J., Echeverri, C.J., Vallee, R.B., and Baas, P.W. 1998. Cytoplasmic dynein and dynactin are required for the transport of microtubules into the axon. J. Cell Biol. 140: 391401.

Aumais, J.P., Tunstead, J.R., McNeil, R.S., Schaar, B.T., McConnell, S.K., Lin, S.H., Clark, G.D., and Yu-Lee, L.Y. 2001. NudC associates with Lis1 and the dynein motor at the leading pole of neurons. J. Neurosci. 21: RC187.

Bai, J., Ramos, R.L., Ackman, J.B., Thomas, A.M., Lee, R.V., and LoTurco, J.J. 2003. RNAi reveals doublecortin is required for radial migration in rat neocortex. Nat. Neurosci. 6: 12771283.

Barkovich, A.J., Koch, T.K., and Carrol, C.L. 1991. The spectrum of lissencephaly: Report of ten patients analyzed by magnetic resonance imaging. Ann. Neurol. 30: 139-146.

Beckwith, S.M. and Morris, N.R. 1995. Cytoplasmic dynein is missing at the tip in NUDG. A nuclear migration mutant in Aspergillus nidulans. Mol. Biol. Cell 6: 5 a.

Bellion, A., Baudoin, J.P., Alvarez, C., Bornens, M., and Metin, C. 2005. Nucleokinesis in tangentially migrating neurons comprises two alternating phases: Forward migration of the Golgi/centrosome associated with centrosome splitting and myosin contraction at the rear. J. Neurosci. 25: 5691-5699.

Burakov, A., Nadezhdina, E., Slepchenko, B., and Rodionov, V. 2003. Centrosome positioning in interphase cells. J. Cell
Biol. 162: 963-969.

Busson, S., Dujardin, D., Moreau, A., Dompierre, J., and De Mey, J.R. 1998. Dynein and dynactin are localized to astral microtubules and at cortical sites in mitotic epithelial cells. Curr. Biol. 8: 541-544.

Coquelle, F.M., Caspi, M., Cordelieres, F.P., Dompierre, J.P., Dujardin, D.L., Koifman, C., Martin, P., Hoogenraad, C.C., Akhmanova, A., Galjart, N., et al. 2002. LIS1, CLIP-170's key to the dynein/dynactin pathway. Mol. Cell. Biol. 22: 3089-3102.

Crome, L. 1956. Pachygyria. J. Pathol. Bacteriol. 71: 335-352.

des Portes, V., Pinard, J.M., Billuart, P., Vinet, M.C., Koulakoff, A., Carrie, A., Gelot, A., Dupuis, E., Motte, J., Berwald-Netter, Y., et al. 1998. A novel CNS gene required for neuronal migration and involved in X-linked subcortical laminar heterotopia and lissencephaly syndrome. Cell 92: 51-61.

Dobyns, W.B. and Truwit, C.L. 1995. Lissencephaly and other malformations of cortical development: 1995 update. Neuropediatrics 26: 132-147.

Dujardin, D.L., Barnhart, L.E., Stehman, S.A., Gomes, E.R., Gundersen, G.G., and Vallee, R.B. 2003. A role for cytoplasmic dynein and LIS1 in directed cell movement. J. Cell Biol. 163: 1205-1211.

Efimov, V.P. and Morris, N.R. 2000. The LIS1-related NUDF protein of Aspergillus nidulans interacts with the coiled-coil domain of the NUDE/RO11 protein. J. Cell Biol. 150: 681688.

Eshel, D., Urrestarauzu, L.A., Vissers, S., Jauniaux, J.C., van Vliet-Reedijk, J.C., Planta, R.J., and Gibbons, I.R. 1993. Cytoplasmic dynein is required for normal nuclear segregation in yeast. Proc. Nat1. Acad. Sci. 90: 11172-11176.

Etienne-Manneville, S. and Hall, A. 2001. Integrin-mediated activation of $\mathrm{Cdc} 42$ controls cell polarity in migrating astrocytes through PKC $\zeta$. Cell 106: 489-498.

Faulkner, N.E., Dujardin, D.L., Tai, C.Y., Vaughan, K.T., O'Connell, C.B., Wang, Y., and Vallee, R.B. 2000. A role for the lissencephaly gene LIS1 in mitosis and cytoplasmic dynein function. Nat. Cell Biol. 2: 784-791.

Feng, Y., Olson, E.C., Stukenberg, P.T., Flanagan, L.A., Kirschner, M.W., and Walsh, C.A. 2000. LIS1 regulates CNS lamination by interacting with $\mathrm{mNudE}$, a central component of the centrosome. Neuron 28: 665-679.

Fleck, M.W., Hirotsune, S., Gambello, M.J., Phillips-Tansey, E., Suares, G., Mervis, R.F., Wynshaw-Boris, A., and McBain, C.J. 2000. Hippocampal abnormalities and enhanced excitability in a murine model of human lissencephaly. J. Neurosci. 20: 2439-2450.

Gambello, M.J., Darling, D.L., Yingling, J., Tanaka, T., Gleeson, J.G., and Wynshaw-Boris, A. 2003. Multiple dose-dependent effects of Lis 1 on cerebral cortical development. J. Neurosci. 23: $1719-1729$.

Gleeson, J.G., Allen, K.M., Fox, J.W., Lamperti, E.D., Berkovic, S., Scheffer, I., Cooper, E.C., Dobyns, W.B., Minnerath, S.R., Ross, M.E., et al. 1998. Doublecortin, a brain-specific gene mutated in human X-linked lissencephaly and double cortex syndrome, encodes a putative signaling protein. Cell 92: 6372.

Gleeson, J.G., Lin, P.T., Flanagan, L.A., and Walsh, C.A. 1999. Doublecortin is a microtubule-associated protein and is expressed widely by migrating neurons. Neuron 23: 257-271.

Gomes, E.R., Jani, S., and Gundersen, G.G. 2005. Nuclear movement regulated by Cdc42, MRCK, myosin, and actin flow establishes MTOC polarization in migrating cells. Cell 121: 451-463.

Gotz, M. and Huttner, W.B. 2005. The cell biology of neurogenesis. Nat. Rev. Mol. Cell Biol. 6: 777-788. 
Han, G., Liu, B., Zhang, J., Zuo, W., Morris, N.R., and Xiang, X. 2001. The Aspergillus cytoplasmic dynein heavy chain and NUDF localize to microtubule ends and affect microtubule dynamics. Curr. Biol. 11: 719-724.

Hattori, M., Adachi, H., Tsujimoto, M., Arai, H., and Inoue, K. 1994. Miller-Dieker lissencephaly gene encodes a subunit of brain platelet-activating factor. Nature 370: 216-218.

Hirotsune, S., Fleck, M.W., Gambello, M.J., Bix, G.J., Chen, A., Clark, G.D., Ledbetter, D.H., McBain, C.J., and WynshawBoris, A. 1998. Graded reduction of Pafah1b1 (Lis1) activity results in neuronal migration defects and early embryonic lethality. Nat. Genet. 19: 333-339.

Kitagawa, M., Umezu, M., Aoki, J., Koizumi, H., Arai, H., and Inoue, K. 2000. Direct association of LIS1, the lissencephaly gene product, with a mammalian homologue of a fungal nuclear distribution protein, rNUDE. FEBS Lett. 479: 57-62.

Koizumi, H., Yamaguchi, N., Hattori, M., Ishikawa, T.O., Aoki, J., Taketo, M.M., Inoue, K., and Arai, H. 2003. Targeted disruption of intracellular type I platelet activating factoracetylhydrolase catalytic subunits causes severe impairment in spermatogenesis. J. Biol. Chem. 278: 12489-12494.

Komarova, Y.A., Akhmanova, A.S., Kojima, S., Galjart, N., and Borisy, G.G. 2002. Cytoplasmic linker proteins promote microtubule rescue in vivo. J. Cell Biol. 159: 589-599.

Kriegstein, A.R. and Noctor, S.C. 2004. Patterns of neuronal migration in the embryonic cortex. Trends Neurosci. 27: 392-399.

Lee, W.L., Oberle, J.R., and Cooper, J.A. 2003. The role of the lissencephaly protein Pacl during nuclear migration in budding yeast. J. Cell Biol. 160: 355-364.

Lei, Y. and Warrior, R. 2000. The Drosophila lissencephaly1 (DLis1) gene is required for nuclear migration. Dev. Biol. 226: 57-72.

Li, Y.Y., Yeh, E., Hays, T., and Bloom, K. 1993. Disruption of mitotic spindle orientation in a yeast dynein mutant. Proc. Natl. Acad. Sci. 90: 10096-10100.

Liang, Y., Yu, W., Li, Y., Yang, Z., Yan, X., Huang, Q., and Zhu, $X$. 2004. Nudel functions in membrane traffic mainly through association with Lis1 and cytoplasmic dynein. I. Cell Biol. 164: 557-566.

Liu, Z., Xie, T., and Steward, R. 1999. Lis1, the Drosophila homolog of a human lissencephaly disease gene, is required for germline cell division and oocyte differentiation. Development 126: 4477-4488.

Lo Nigro, C., Chong, C.S., Smith, A.C., Dobyns, W.B., Carrozzo, R., and Ledbetter, D.H. 1997. Point mutations and an intragenic deletion in LIS1, the lissencephaly causative gene in isolated lissencephaly sequence and Miller-Dieker syndrome. Hum. Mol. Genet. 6: 157-164.

Mesngon, M.T., Tarricone, C., Hebbar, S., Guillotte, A.M., Schmitt, E.W., Lanier, L., Musacchio, A., King, S.J., and Smith, D.S. 2006. Regulation of cytoplasmic dynein ATPase by Lis1. J. Neurosci. 26: 2132-2139.

Morris, S.M., Albrecht, U., Reiner, O., Eichele, G., and Yu-Lee, L.Y. 1998. The lissencephaly gene product Lis1, a protein involved in neuronal migration, interacts with a nuclear movement protein, NudC. Curr. Biol. 8: 603-606.

Morris, J.A., Kandpal, G., Ma, L., and Austin, C.P. 2003. DISC1 (Disrupted-In-Schizophrenia 1) is a centrosome-associated protein that interacts with MAP1A, MIPT3, ATF4/5 and NUDEL: Regulation and loss of interaction with mutation. Hum. Mol. Genet. 12: 1591-1608.

Niethammer, M., Smith, D.S., Ayala, R., Peng, J., Ko, J., Lee, M.S., Morabito, M., and Tsai, L.H. 2000. NUDEL is a novel Cdk5 substrate that associates with LIS1 and cytoplasmic dynein. Neuron 28: 697-711.
Noctor, S.C., Martinez-Cerdeno, V., Ivic, L., and Kriegstein, A.R. 2004. Cortical neurons arise in symmetric and asymmetric division zones and migrate through specific phases. Nat. Neurosci. 7: 136-144.

Palazzo, A.F., Joseph, H.L., Chen, Y.J., Dujardin, D.L., Alberts, A.S., Pfister, K.K., Vallee, R.B., and Gundersen, G.G. 2001. Cdc42, dynein, and dynactin regulate MTOC reorientation independent of Rho-regulated microtubule stabilization. Curr. Biol. 11: 1536-1541.

Paschal, B.M. and Vallee, R.B. 1987. Retrograde transport by the microtubule-associated protein MAP 1C. Nature 330: 181183.

Paschal, B.M., King, S.M., Moss, A.G., Collins, C.A., Vallee, R.B., and Witman, G.B. 1987. Isolated flagellar outer arm dynein translocates brain microtubules in vitro. Nature 330: 672-674.

Reiner, O., Carrozzo, R., Shen, Y., Wehnert, M., Faustinella, F., Dobyns, W.B., Caskey, C.T., and Ledbetter, D.H. 1993. Isolation of a Miller-Dieker lissencephaly gene containing G protein b-subunit-like repeats. Nature 364: 717-721.

Ross, M.E. 2002. Brain malformations, epilepsy, and infantile spasms. Int. Rev. Neurobiol. 49: 333-352.

Sapir, T., Elbaum, M., and Reiner, O. 1997. Reduction of microtubule catastrophe events by LIS1, platelet-activating factor acetylhydrolase subunit. EMBO J. 16: 6977-6984.

Sasaki, S., Shionoya, A., Ishida, M., Gambello, M.J., Yingling, J., Wynshaw-Boris, A., and Hirotsune, S. 2000. A LIS1/NUDEL/ cytoplasmic dynein heavy chain complex in the developing and adult nervous system. Neuron 28: 681-696.

Schaar, B.T. and McConnell, S.K. 2005. Cytoskeletal coordination during neuronal migration. Proc. Natl. Acad. Sci. 102: 13652-13657.

Shu, T., Ayala, R., Nguyen, M.D., Xie, Z., Gleeson, J.G., and Tsai, L.H. 2004. Ndell operates in a common pathway with LIS1 and cytoplasmic dynein to regulate cortical neuronal positioning. Neuron 44: 263-277.

Smith, D.S., Niethammer, M., Ayala, R., Zhou, Y., Gambello, M.J., Wynshaw-Boris, A., and Tsai, L.H. 2000. Regulation of cytoplasmic dynein behaviour and microtubule organization by mammalian Lis1. Nat. Cell Biol. 2: 767-775.

Solecki, D.J., Model, L., Gaetz, J., Kapoor, T.M., and Hatten, M.E. 2004. Par6 $\alpha$ signaling controls glial-guided neuronal migration. Nat. Neurosci. 7: 1195-1203.

Stewart, R.M., Richman, D.P., and Caviness Jr., V.S. 1975. Lissencephaly and Pachygyria: An architectonic and topographical analysis. Acta Neuropathol. (Berl.) 31: 1-12.

Swan, A., Nguyen, T., and Suter, B. 1999. Drosophila Lissencephaly-1 functions with Bic-D and dynein in oocyte determination and nuclear positioning. Nat. Cell Biol. 1: 444449.

Sweeney, K.J., Clark, G.D., Prokscha, A., Dobyns, W.B., and Eichele, G. 2000. Lissencephaly associated mutations suggest a requirement for the PAFAH1B heterotrimeric complex in brain development. Mech. Dev. 92: 263-271.

Tai, C.Y., Dujardin, D.L., Faulkner, N.E., and Vallee, R.B. 2002. Role of dynein, dynactin, and CLIP-170 interactions in LIS1 kinetochore function. J. Cell Biol. 156: 959-968.

Tanaka, T., Serneo, F.F., Higgins, C., Gambello, M.J., WynshawBoris, A., and Gleeson, J.G. 2004. Lis1 and doublecortin function with dynein to mediate coupling of the nucleus to the centrosome in neuronal migration. J. Cell Biol. 165: 709721.

Toyo-oka, K., Shionoya, A., Gambello, M.J., Cardoso, C., Leventer, R., Ward, H.L., Ayala, R., Tsai, L.H., Dobyns, W., Ledbetter, D., et al. 2003. $14-3-3 \varepsilon$ is important for neuronal migration by binding to NUDEL: A molecular explanation 
for Miller-Dieker syndrome. Nat. Genet. 34: 274-285.

Tsai, J.W., Chen, Y., Kriegstein, A.R., and Vallee, R.B. 2005. LIS1 RNA interference blocks neural stem cell division, morphogenesis, and motility at multiple stages. J. Cell Biol. 170: 935-945.

Uetake, Y., Terada, Y., Matuliene, J., and Kuriyama, R. 2004. Interaction of Cep135 with a p50 dynactin subunit in mammalian centrosomes. Cell Motil. Cytoskeleton 58: 53-66.

Vallee, R.B. and Stehman, S.A. 2005. How dynein helps the cell find its center: A servomechanical model. Trends Cell Biol. 15: $288-294$.

Varma, D., Dujardin, D.L., Stehman, S.A., and Vallee, R.B. 2006. Role of the kinetochore/cell cycle checkpoint protein ZW10 in interphase cytoplasmic dynein function. J. Cell Biol. 172: 655-662.

Xiang, X., Osmani, A.H., Osmani, S.A., Xin, M., and Morris, N.R. 1995. NudF, a nuclear migration gene in Aspergillus nidulans, is similar to the human LIS- 1 gene required for neuronal migration. Mol. Biol. Cell 6: 297-310.

Yan, W., Assadi, A.H., Wynshaw-Boris, A., Eichele, G., Matzuk, M.M., and Clark, G.D. 2003. Previously uncharacterized roles of platelet-activating factor acetylhydrolase $1 \mathrm{~b}$ complex in mouse spermatogenesis. Proc. Natl. Acad. Sci. 100: 7189-7194. 


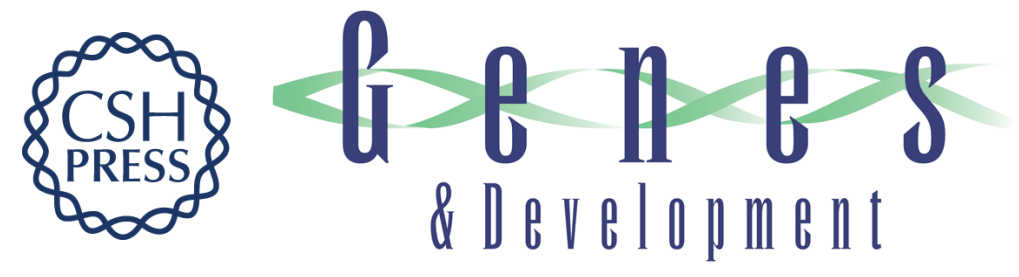

\section{The cellular roles of the lissencephaly gene LIS1, and what they tell us aboutbrain development}

Richard B. Vallee and Jin-Wu Tsai

Genes Dev. 2006, 20:

Access the most recent version at doi:10.1101/gad.1417206

References This article cites 65 articles, 25 of which can be accessed free at: http://genesdev.cshlp.org/content/20/11/1384.full.html\#ref-list-1

License

Email Alerting

Receive free email alerts when new articles cite this article - sign up in the box at the top Service right corner of the article or click here.

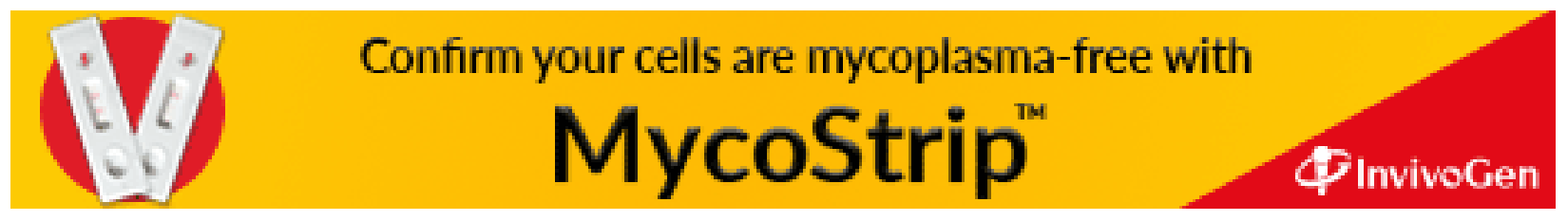

